\title{
An Identification of the Connections of Quillen and Beilinson-Schechtman
}

\author{
Yue Lin L. Tong, ${ }^{1 \star}$ I-Hsun Tsai ${ }^{2 \star \star}$ \\ 1 Purdue University, West Lafayette, IN 47907, USA \\ 2 National Taiwan University, Taipei, Taiwan
}

Received: 20 January 1993

\begin{abstract}
Given a family of Riemann surfaces and a holomorphic vector bundle Beilinson and Schechtman construct a canonical connection on the associated determinant bundle. We prove the conjecture which states that their connection coincides with the Quillen connection. This is done by reducing to the case where $\bar{\partial}$ along fibers are invertible. Both connection forms become more accessible in this case.
\end{abstract}

\section{Introduction}

Let $\pi: X \rightarrow S$ be the parametrization of a family of compact Riemann surfaces, $E \rightarrow X$ a holomorphic vector bundle, and $\lambda_{E}=\operatorname{det}\left(R \pi_{*} E\right)$ the determinant bundle over $S$. Given $C^{\infty}$ connections on $T_{X / S}$ and $E$, not necessarily arising from metrics, Beilinson and Schechtman [BS, §5] construct a formal parametrix $p(z, \zeta)$ for $\bar{\partial}_{z}$ ( $\bar{\partial}$ along the fibers) and using $p$ they derive a $(1,0)$ connection $\nabla_{\mathrm{BS}}$ on $\lambda_{E}$. These are given by local formulas in terms of $\nabla_{T_{X / S}}$ and $\nabla_{E}$ and fiber integrals. Local calculations show that the $(1,1)$ curvature $\bar{\partial}_{S} \nabla_{\mathrm{BS}}$ is equal as a differential form to the fiber integral prescribed by the Grothendieck Riemann Roch formula. The only nonelementary part of the connection $\nabla_{\mathbf{B S}}$ is in identifying it with splittings of complexes on $X$, which depends on the relative duality theorem.

On $\lambda_{E}^{-1}$ of course there is the well known Quillen metric and the associated Quillen connection $\nabla_{\mathrm{Q}}$ determined by metrics on $T_{X / S}$ and $E$. For locally Kähler families of compact complex manifolds the form level Grothendieck Riemann Roch for curvature of $\nabla_{\mathrm{Q}}$ is known [BF, BGS]. Beilinson-Schechtman remark in [BS, 5.6]: "It seems very probable" that when $\nabla_{E}, \nabla_{T_{X / S}}$ arise from hermitian metrics on $E, T_{X / S}$, then $\nabla_{\mathrm{BS}}$ is just the connection associated to the Quillen metric for $\lambda_{E}$.

\footnotetext{
* Supported in part by N.S.F. Grant No. DMS-9201022

$\star \star$ Supported in part by National Science Council of Republic of China Grant No. NSC 82-0208-M-002-125-T, and NSERC of Canada Grant No. OGP 0121883
} 
Since $\nabla_{\mathrm{Q}}$ determines uniquely a connection on $\lambda_{E}$ which we denote by $-\nabla_{\mathrm{Q}}$, Beilinson-Schechtman's conjecture is the identification $\nabla_{\mathrm{BS}}=-\nabla_{\mathrm{Q}}$. The Quillen metric and $\nabla_{\mathrm{Q}}$, being defined in terms of $\bar{\partial}$ torsion, are highly analytical in nature; on the other hand $\nabla_{\mathrm{BS}}$ acting through the Kodaira-Spencer class and formal parametrics has a rather geometric and sheaf cohomological flavor. It is obviously of interest then to unify the two seemingly disparate objects.

The purpose of this paper is to give such an identification. By adding to $E$ a vector bundle of the opposite index along fibers we may reduce to the case where the index of $E$ is zero. Under this hypothesis it is easy to further reduce to families in which $\bar{\partial}_{z}$ is invertible over a dense open subset of $S$, this being so in the moduli space. In this case there is a canonical holomorphic section of $\lambda_{E}^{-1}$ with respect to which the connection one form of $\nabla_{\mathrm{Q}}$ has a striking interpretation as the heat kernel renormalization of $\operatorname{Tr}_{s}\left[D^{-1}(\tilde{\nabla} D)\right][Q, B F]$ :

$$
\lim _{t \rightarrow 0} \operatorname{Tr}_{s}\left[e^{-t D^{2}} D^{-1}(\tilde{\nabla} D)\right],
$$

where $D=\bar{\partial}_{z}+\bar{\partial}_{z}^{*}$ and $\tilde{\nabla}$ is the connection on an infinite dimensional bundle induced by $\nabla_{E}$ and $\nabla_{T_{X / S}}$. In general $\nabla_{\mathrm{BS}, v}$ is represented by a cohomology class on $X$. In the situation where $\bar{\partial}_{z}$ is invertible there exists a canonical integrable connection $\nabla$ on $\lambda_{E}$, and if the metric on $T_{X / S}$ is locally Kähler then the difference of $\nabla_{\mathrm{BS}}$ and $\nabla$ is given by the fiber integral of a smooth form:

$$
\nabla_{\mathbf{B S}, v}-\nabla_{v}=\int_{X / S}\left(p^{-1}-G\right)\left(\bar{\partial}_{z} \tilde{v}\right)
$$

where $\tilde{v} \in \mathscr{T}_{X}$ is the horizontal lift of $v \in \mathscr{T}_{S}$ with respect to the metric, so $\bar{\partial}_{z} \tilde{v}$ is a representative of the Kodaira-Spencer class of $v, p^{-1}\left(\bar{\partial}_{z} \tilde{v}\right)=\left(\bar{\partial}_{z} \tilde{v}(z)\right) p(z, \zeta)$ and $G$ is the kernel function of $\bar{\partial}_{z}^{-1}$.

The formulas (1) and (2) readily reduce the identification problem $\nabla_{\mathrm{BS}}=-\nabla_{\mathrm{Q}}$ to proving the vanishing statement:

$$
\lim _{t \rightarrow 0} \operatorname{Tr}\left[e^{-t \Delta} p^{-1}\left(\overline{\partial_{z}} \tilde{v}\right)\right]=0 .
$$

This is an analogue (extended to first derivatives) of the vanishing result of Quillen $[Q, \S 5]$. This is the most technical part of the proof, and it relies on the expansion of $e^{-t \Delta}$ and $p(z, \zeta)$ in local geodesic coordinates in Sect. 4. Having proved $\nabla_{\mathrm{BS}}=-\nabla_{\mathrm{Q}}$ for a locally Kähler metric we use variation formulas to show $\nabla_{\mathrm{BS}}=-\nabla_{\mathrm{Q}}$ for any hermitian metric on $T_{X / S}$. For two metrics $g$ and $g^{\prime}$ on $T_{X / S}$ and the associated connections $\nabla_{\mathrm{Q}}, \nabla_{\mathrm{Q}}^{\prime}$ the difference $\nabla_{\mathrm{Q}}^{\prime}-\nabla_{\mathrm{Q}}$ is given in terms of Bott-Chern forms [BGS III]; we show by a direct calculation in Sect. 2 that we have precisely $\nabla_{\mathrm{BS}}^{\prime}-\nabla_{\mathrm{BS}}=-\left(\nabla_{\mathrm{Q}}^{\prime}-\nabla_{\mathrm{Q}}\right)$.

We may interpret this identification as saying that the Quillen connection which depends on data of metrics, has a canonical extension to a connection which depends on data of connections. This leads to some natural questions which we hope to address elsewhere. At present the construction of the Beilinson-Schechtman parametrix $p$ is limited to families of Riemann surfaces. There is a Cech cohomological construction of an analogous $p$ in higher relative dimensions which also yields a connection $\nabla_{p}$ on $\lambda_{E}$ and a form level Grothendieck Riemann Roch without any Kähler hypothesis [T]. However it will not be possible to identify $\nabla_{p}$ and $\nabla_{\mathrm{Q}}$ for the simple reason that the Chern forms appearing in the fiber integral in $[\mathrm{T}]$ for $\bar{\partial}_{s} \nabla_{p}$ are the Atiyah-Chern forms which are different from the standard 
Chern forms defined from metrics. The Atiyah-Chern forms for example are $\bar{\partial}$ closed but in general not $d$ closed. Thus the fiber integrals giving the curvatures of $\nabla_{p}$ and $\nabla_{\mathrm{Q}}$ are different.

We thank J.M. Bismut, J.B. Bost, H. Donnelly, L. Lempert, and C. Soulé for helpful discussions.

\section{The Beilinson-Schechtman Connection}

Let $\pi: X \rightarrow S$ be a proper smooth map of complex manifolds of relative dimension one, $E \rightarrow X$ a holomorphic vector bundle and $\lambda_{E}=\operatorname{det} R \pi_{*} E$ the determinant bundle associated to the direct image complex. The Atiyah algebra of $\lambda_{E}$ is the sheaf of local connections of $\lambda_{E}$ which in this case is an extension of the tangent sheaf $\mathscr{T}_{S}$ by the structure sheaf $\mathcal{O}_{S}$ :

$$
0 \rightarrow \mathcal{O}_{S} \rightarrow \mathscr{A}_{\lambda_{E}} \rightarrow \mathscr{T}_{S} \rightarrow 0 .
$$

In [BS] Beilinson and Schechtman construct a differential graded Lie super algebra ${ }^{\operatorname{tr}} \mathscr{A}_{E}^{\cdot}$ on $X$ whose direct image cohomology $R^{0} \pi_{*}\left({ }^{\text {tr }} \mathscr{A}_{E}^{\cdot}\right)$ is canonically isomorphic to $\mathscr{A}_{\lambda_{E}}$.

Let $C_{S}$ be the sheaf of germs of $C^{\infty}$ functions on $S$ then a $C^{\infty}(1,0)$ connection on $\lambda_{E}$ is a splitting of the exact sequence:

$$
0 \rightarrow C_{S} \rightarrow C \mathscr{A}_{\lambda_{E}} \rightarrow \mathscr{T}_{S} \rightarrow 0 .
$$

To represent (1.1) as cohomology of complexes on $X$ let $\mathscr{A}_{E, \pi} \subset \mathscr{A}_{E}$ be the subalgebra whose image in the natural map $\varepsilon: \mathscr{A}_{E} \rightarrow \mathscr{T}_{X}$ equals $\mathscr{T}_{\pi}$, the projectable vector fields. Further let $\mathscr{A}_{E / S} \subset \mathscr{A}_{E, \pi}$ be the ideal which differentiates in the fiber directions. $C^{\text {tr }} \mathscr{A}_{E}^{-1}$ is the push forward of ${ }^{\text {tr }} \mathscr{A}_{E}^{-1}$ by the map $\Omega_{X / S} \rightarrow \Omega_{X / S}{ }_{X / S}$, where $\Omega_{X / S}$ is the complex of holomorphic relative forms and $\Omega^{\prime, \cdot}=\Omega_{\dot{X} / S}$ the complex of bigraded $C^{\infty}$ relative forms. In local coordinates near $\Delta$ in $X \times{ }_{S} X$ the elements of $C^{\operatorname{tr}} \mathscr{A}_{E}^{-1}$ are given by:

$$
\left\{\frac{a(z)}{(\zeta-z)^{2}}+\frac{B(z)}{\zeta-z}+c(z)\right\} d \zeta,
$$

where $a(z)$ and $B(z)$ are holomorphic $\left(B(z)\right.$ takes value in $\left.E \otimes E^{*}\right)$ and $c(z)$ is $C^{\infty}$. Taking residues along $\Delta$ defines a map $R: C^{\text {tr }} \mathscr{A}_{E}^{-1} \rightarrow \mathscr{A}_{E / S}$ and there arise maps of complexes

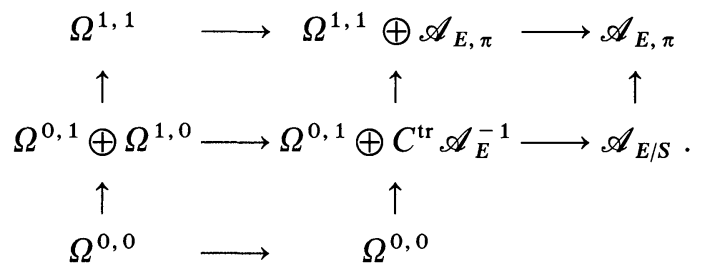

The vertical complexes in (1.2) are assigned degree zero in their top degrees, and $R^{0} \pi_{*}$ applied to (1.2) is canonically isomorphic to (1.1).

Suppose given $C^{\infty}$ connections $\nabla_{T}$ and $\nabla_{E}$ on $T=T_{X / S}$ and $E$ respectively, the Beilinson-Schechtman connection $\nabla_{\mathrm{BS}}=R^{0} \pi_{*}\left(p^{\circ}\right)$, where $p^{*}$ is a splitting of (1.2). Namely $\nabla_{T}$ and $\nabla_{E}$ determine a formal parametrix $p(z, \zeta) \in C E \otimes E^{\prime}(\Delta)$, 
$E^{\prime}=E^{*} \otimes \Omega_{X / S}^{1}[\mathrm{BS}, \S 5.3]$. Thus $p(z, \zeta)$ is $C^{\infty}$ in $z$, holomorphic in $\zeta$ with a first order pole along $\Delta$, and $R P=1 \in \mathscr{A}_{E / S}$. For $u \in \mathscr{A}_{E / S}$, set

$$
p^{-1}(u)=(0, \beta u(z) p(z, \zeta)) \in \Omega^{0,1} \oplus C^{\operatorname{tr}} \mathscr{A}_{E}^{-1},
$$

where $\beta$ is $\operatorname{tr} \circ \Delta^{*}$ applied to nonsingular terms of $u(z) p(z, \zeta)$ and leaves singular terms unchanged. $\bar{\partial}_{X} p^{-1}$ naturally extends to a sheaf map $\mathscr{A}_{E, \pi} \rightarrow \Omega_{X / S}^{1,0} \otimes \Omega_{X}^{0,1}$, and

$$
p^{0}(v)=\left(\left(\bar{\partial}_{z} p^{-1}\right)(v), v\right) \in \Omega_{X / S}^{1,1} \oplus \mathscr{A}_{E, \pi},
$$

where $\bar{\partial}_{z}$ denotes $\bar{\partial}$ along the fibers.

In local coordinates suppose the $(1,0)$ components of $\nabla_{T}$ and $\nabla_{E}$ along the fiber directions are

$$
\nabla_{T, \frac{\partial}{\partial z}}=\partial_{z}+\Phi_{T} d z, \quad \nabla_{E, \frac{\partial}{\partial z}}=\partial_{z}+\Phi_{E} d z
$$

The components of curvature forms valued in $\Omega_{X}^{1,1}$ are denoted by $C\left(\nabla_{T}\right)$ and $C\left(\nabla_{E}\right)$, and their projections into $\Omega_{X / S}^{1,1}$ are denoted $\tilde{C}\left(\nabla_{T}\right)=\bar{\partial}_{z} \Phi_{T} d z$ and $\tilde{C}\left(\nabla_{E}\right)=\bar{\partial}_{z} \Phi_{E} d z$. Furthermore, $c_{1}\left(\nabla_{E}\right)=\frac{i}{2 \pi} \operatorname{tr} C\left(\nabla_{E}\right), \tilde{c}_{1}\left(\nabla_{E}\right)=\frac{i}{2 \pi} \operatorname{tr} \tilde{C}\left(\nabla_{E}\right)$ and similarly for $\nabla_{T} . \omega=\omega_{X / S}$ is the relative canonical bundle and $\nabla_{T}$ induces $\nabla_{\omega}$ with $\nabla_{\omega, \frac{\partial}{\partial z}}=\partial_{z}-\Phi_{T} d z$. To write down $p^{\cdot}$ in local coordinates let $u=a \frac{\partial}{\partial z}+B \in \mathscr{A}_{E / S}$ and define $\pi_{\nabla_{E}}: \mathscr{A}_{E, \pi} \rightarrow \operatorname{End}(E) \otimes \Omega^{0,0}$ by

$$
\pi_{\nabla_{E}}(v)=v-\nabla_{E, \varepsilon(v)} .
$$

We now have

$$
\begin{gathered}
p(z, \zeta)=\frac{1}{2 \pi i}\left\{\frac{1}{\zeta-z}+\sum_{i \geqq 0} A_{i}(z)(\zeta-z)^{i}\right\} d \zeta, \\
A_{0}(z)=\frac{1}{2} \Phi_{T}(z)+\Phi_{E}(z), \\
A_{1}(z)=\frac{1}{6} \Phi_{T}^{\prime}(z)-\frac{1}{12} \Phi_{T}^{2}(z)-\frac{1}{2} \Phi_{T}(z) \Phi_{E}(z)+\frac{1}{2} \Phi_{E}^{\prime}(z)-\frac{1}{2} \Phi_{E}^{2}(z),
\end{gathered}
$$

and

$$
\begin{aligned}
p^{-1}(u)= & \frac{1}{2 \pi i}\left\{\frac{a}{(\zeta-z)^{2}}+\frac{B}{\zeta-z}+\operatorname{tr}\left[B A_{0}+a A_{1}\right]\right\} d \zeta, \\
p^{0}(v)= & \left\{-\nabla_{\omega, \varepsilon(v)}\left(\frac{m}{6} \tilde{c}_{1}\left(\nabla_{T}\right)+\frac{1}{2} \tilde{c}_{1}\left(\nabla_{E}\right)\right)\right. \\
& \left.-\operatorname{tr}\left[\pi_{\nabla_{E}}(v)\left(\frac{1}{2} \tilde{C}\left(\nabla_{T}\right)+\tilde{C}\left(\nabla_{E}\right)\right)\right], v\right\}
\end{aligned}
$$

(the minus signs in $p^{0}(v)$ come from shifting $\frac{1}{2 \pi i}$ to $\frac{i}{2 \pi}$ in definition of $c_{1}$ ). 
To calculate the action $R^{0} \pi_{*}(p)$ we consider first resolutions of (1.2) by Cech complexes. Let $\mathscr{U}$ be an acyclic covering of $\pi^{-1}(U)$, where $U \subset S$ is a sufficiently small open set and $\rho$ a partition of 1 subordinate to $\mathscr{U}$. We have

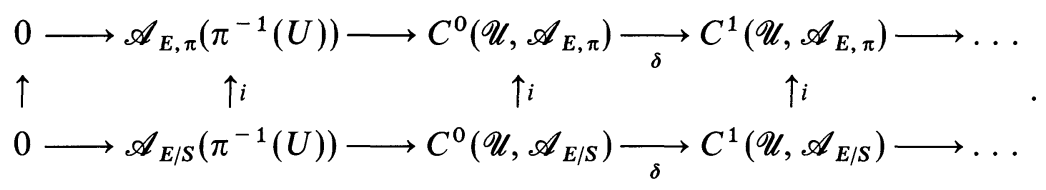

$R^{0} \pi_{*}\left(\mathscr{A}_{E}^{*}\right)(U)$ is represented by

$$
\left\{(v, u) \in C^{0}\left(\mathscr{U}, \mathscr{A}_{E, \pi}\right) \oplus C^{1}\left(\mathscr{U}, \mathscr{A}_{E / S}\right),-\delta v+i u=0, \delta u=0\right\}
$$

modulo the coboundaries $(i, \delta) C^{0}\left(\mathscr{U}, \mathscr{A}_{E / S}\right)$. Let

$$
\begin{aligned}
& \rho: C^{0}\left(\mathscr{U}, \mathscr{A}_{E, \pi}\right) \rightarrow C \mathscr{A}_{E, \pi}\left(\pi^{-1}(U)\right):=\left(\mathscr{A}_{E, \pi} \otimes \Omega^{0,0}\right)\left(\pi^{-1}(U)\right), \\
& \rho: C^{1}\left(\mathscr{U}, \mathscr{A}_{E / S}\right) \rightarrow \mathscr{A}_{E / S} \otimes \Omega^{0,1}\left(\pi^{-1}(U)\right)
\end{aligned}
$$

be given by

$$
\rho\left(c^{0}\right)=\sum_{\alpha} \rho_{\alpha} c_{\alpha}^{0}, \quad \rho\left(c^{1}\right)=\sum_{\alpha, \beta} \rho_{\beta} \bar{\partial} \rho_{\alpha} c_{\alpha \beta}^{1} .
$$

Then $\rho$ defines a quasi-isomorphism on total complexes which at degree zero is:

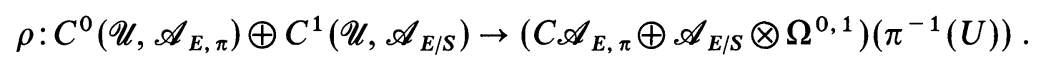

Let $v \in \mathscr{T}_{S}(U)$ be a vector field and $\{v\} \in C^{0}\left(\mathscr{U}, \mathscr{A}_{E, \pi}\right)$ be local lifts, then $\{\delta v\} \in$ $C^{1}\left(\mathscr{U}, \mathscr{A}_{E / S}\right)$ and it is easy to see that if $\tilde{v}=\rho\{v\}$ (cf. $[\mathrm{TT}, \S 1]$ )

$$
\rho(\{v\} \oplus\{\delta v\})=\tilde{v} \oplus \bar{\partial}_{z} \tilde{v} .
$$

We may similarly form Cech resolutions of

$$
\begin{gathered}
\Omega^{1,1} \oplus \mathscr{A}_{E, \pi} \\
\uparrow \\
\Omega^{0,1} \oplus C^{\operatorname{tr}} \mathscr{A}_{E}^{-1},
\end{gathered}
$$

and (1.6) defines quasi-isomorphisms on the corresponding total complexes. Now the advantage of Cech complexes is that the sheaf level splitting (1.4) extends immediately to a chain map of the bicomplex in (1.5) to that of (1.8). We thus have a chain map of the total complexes (associated to Cech complexes) and via $\rho$ a splitting of the following diagram of complexes (of sections over $\pi^{-1}(U)$ ):

$$
\begin{gathered}
C \mathscr{A}_{E, \pi} \oplus \Omega^{1,1} \oplus^{\operatorname{tr}} \mathscr{A}_{E}^{-1} \otimes \Omega^{0,1} \stackrel{\left(\begin{array}{ll}
1 & 0 \\
0 & 0 \\
0 & R
\end{array}\right)}{\longrightarrow} C \mathscr{A}_{E, \pi} \oplus \mathscr{A}_{E / S} \otimes \Omega^{0,1} \\
\uparrow\left(\begin{array}{ccc}
0 & \partial_{z} & 0 \\
R & q \bar{\partial}_{z} & -\bar{\partial}_{z}
\end{array}\right) \\
\Omega^{0,1} \oplus^{\operatorname{tr}} \mathscr{A}_{E}^{-1} \otimes \Omega^{0,0} \stackrel{\left(\begin{array}{l}
0 \\
R
\end{array}\right)}{\longrightarrow} \\
\mathscr{A}_{E / S} \otimes \Omega^{0,0},
\end{gathered}
$$


where $q:^{\text {tr }} \mathscr{A}_{E}^{-1} \otimes \Omega^{0,1} \rightarrow \Omega^{1,1}$ is the projection given by $q=1-p^{-1} R$. It is straightforward to check that the splitting of (1.9) is given by the maps

$$
\left(0, p^{-1}\right) \text { and }\left(\begin{array}{ccc}
1 & p^{0} & 0 \\
0 & 0 & p^{-1}
\end{array}\right) \text {. }
$$

Applying the splitting to the element in (1.7) one obtains

$$
\tilde{v}+p^{0}(\tilde{v})+p^{-1}\left(\bar{\partial}_{z} \tilde{v}\right) .
$$

The cohomology class of this element in the left column of (1.9) represents the action of the Beilinson-Schechtman connection:

$$
\nabla_{\mathbf{B S}, v}=\left[\tilde{v}+p^{-1}\left(\bar{\partial}_{z} \tilde{v}\right)+p^{0}(\tilde{v})\right] .
$$

\section{Comparison of Variation Formulas}

Let $g$ be a hermitian matrix on $T_{X / S}$ and $h$ a hermitian metric on $E$. In the present case of relative dimension one $g$ induces a Kähler metric on the fibers. These determine a Quillen metric \|\|$_{\mathrm{Q}}$ on $\lambda_{E}^{-1}$ and the corresponding Quillen connection $\nabla_{\mathrm{Q}}$. The metrics $g$ and $h$ also determine $(1,0)$ hermitian connections $\nabla_{T}$ and $\nabla_{E}$, and from Sect. 1 we obtain the connection $\nabla_{\mathrm{BS}}$ on $\lambda_{E}$. In this section we compare the variation formulas for $\nabla_{\mathrm{Q}}$ and $\nabla_{\mathrm{BS}}$ when $g$ is changed to a metric $g_{1}$.

Let $g_{1}=e^{\varphi} g$, where $\varphi$ is a $C^{\infty}$ function on $X$ and let $\nabla_{Q}^{1}$ be the Quillen connection associated to $\left(g_{1}, h\right)$. By [BGS III, Th. 1.23].

$$
\begin{aligned}
\nabla_{\mathrm{Q}}^{1}-\nabla_{\mathrm{Q}} & =-\partial_{S}\left[\int_{X / S} \tilde{T} d\left(g, g_{1}\right) \operatorname{ch}(h)\right]_{0} \\
& =-\int_{X / S} \partial\left[\tilde{T} d\left(g, g_{1}\right) \operatorname{ch}(h)\right]_{1},
\end{aligned}
$$

where []$_{i}$ denotes the component of degree $(i, i)$. Let $g_{t}=e^{t \phi} g$ be the family connecting $g$ to $g_{1}$, then

$$
\begin{aligned}
\partial \log g_{t} & =\partial \log g+t \partial \phi \\
\frac{d}{d t}\left(\partial \log g_{t}\right) & =\partial \phi \\
\frac{i}{2 \pi} \bar{\partial} \partial \log g_{t} & =c_{1}\left(\nabla_{T}\right)+t \frac{i}{2 \pi} \bar{\partial} \partial \phi .
\end{aligned}
$$

In the present case

$$
[T d(g) \operatorname{ch}(h)]_{2}=\frac{m}{12} c_{1}\left(\nabla_{T}\right)^{2}+\frac{1}{2} c_{1}\left(\nabla_{T}\right) c_{1}\left(\nabla_{E}\right)+c h_{2}\left(\nabla_{E}\right)
$$

\footnotetext{
${ }^{1}$ Added in proof: There is a simpler justification that (1.10) represents the action of a connection This will be discussed elsewhere.
} 
where $m=r k(E)$. Then using the Bott-Chern formulas [Bt C, 3.15, BGSI, Th. $1.27]$, one gets readily

$$
\nabla_{Q}^{1}-\nabla_{Q}=-\int_{X / S}\left\{\frac{m}{6} \partial \phi \wedge c_{1}\left(\nabla_{T}\right)+\frac{m}{12} \partial \phi \wedge \frac{i}{2 \pi} \bar{\partial} \partial \phi+\frac{1}{2} \partial \phi \wedge c_{1}\left(\nabla_{E}\right)\right\} .
$$

To compute the variation formula for the Beilinson-Schechtman connection let $p_{1}^{0}$, $p_{1}^{-1}$ denote the maps (1.4) computed with respect to the connections $\nabla_{T}^{1}=\nabla_{T}+\partial \phi$ and $\nabla_{E}$. Then by $(1.10)$

$$
\nabla_{\mathrm{BS}, v}^{1}-\nabla_{\mathrm{BS}, v}=\left[p_{1}^{-1}\left(\bar{\partial}_{z} \tilde{v}\right)-p^{-1}\left(\bar{\partial}_{z} \tilde{v}\right)+p_{1}^{0}(\tilde{v})-p^{0}(\tilde{v})\right]
$$

This is a cohomology class of a smooth form in $\Omega_{X / S}^{1,1}$ and hence the class is uniquely determined by the fiber integral of the form. Therefore,

$$
\nabla_{\mathrm{BS}, v}^{1}-\nabla_{\mathrm{BS}, v}=\int_{X / \mathbf{S}}\left(p_{1}^{-1}-p^{-1}\right)\left(\bar{\partial}_{z} \tilde{v}\right)+\left(p_{1}^{0}-p^{0}\right)(\tilde{v}) .
$$

Proposition 2.1. $\nabla_{\mathrm{BS}}^{1}-\nabla_{\mathrm{BS}}=-\left(\nabla_{\mathrm{Q}}^{1}-\nabla_{\mathrm{Q}}\right)$.

Proof. The integral (2.4) is independent of the choice of $\tilde{v}$ which lifts $v$. In local coordinates let $v=\frac{\partial}{\partial s}$ be a germ of $\mathscr{T}_{s}$ and let $v_{0}=\frac{\partial}{\partial s}+a \frac{\partial}{\partial z}$ be a lift to $C^{\infty}\left(\mathscr{T}_{\pi}\right)$. We then set

$$
\tilde{v}=\nabla_{E, v_{0}}=\frac{\partial}{\partial s}+a \frac{\partial}{\partial z}+B \in C \mathscr{A}_{E, \pi} .
$$

With this choice

$$
\pi_{\nabla_{E}}(\tilde{v})=\tilde{v}-\nabla_{E, \varepsilon(\tilde{v})}=0
$$

We also have

$$
\bar{\partial}_{z} v_{0}=\left(\bar{\partial}_{z} a\right) \frac{\partial}{\partial z}, \quad \bar{\partial}_{z} \tilde{v}=\left(\bar{\partial}_{z} a\right) \frac{\partial}{\partial z}+\bar{\partial}_{z} B
$$

and differentiating (2.6): ( $\rfloor$ denotes contraction)

$$
\left.\bar{\partial}_{z} \tilde{v}-\nabla_{E, \bar{\partial}_{z} v_{0}}=-v_{0}\right\rfloor C(E) .
$$

We now expand by (1.4)

$$
\begin{aligned}
\left(p_{1}^{-1}-p^{-1}\right)\left(\bar{\partial}_{z} \tilde{v}\right)= & \frac{1}{2 \pi i} \operatorname{tr}\left\{\bar{\partial}_{z} B\left(\frac{1}{2} \phi^{\prime}\right)\right. \\
& \left.+\bar{\partial}_{z} a\left(\frac{1}{6} \phi^{\prime \prime}-\frac{1}{12}\left(2 \Phi_{T}+\phi^{\prime}\right) \phi^{\prime}-\frac{1}{2} \Phi_{E} \phi^{\prime}\right)\right\} d z \\
= & \frac{i}{2 \pi}\left\{-\frac{m}{6} \nabla_{\omega, \bar{\partial}_{z} v_{0}}\left(\partial_{z} \phi\right) \cdots(\mathrm{i})\right. \\
& \left.+\frac{m}{12}\left(\bar{\partial}_{z} v_{0}\right\rfloor \partial \phi\right) \partial_{z} \phi \cdots \text { (ii) } \\
& \left.-\frac{1}{2} \operatorname{tr}\left(\bar{\partial}_{z} \tilde{v}-\nabla_{E, \bar{\partial}_{z} v_{0}}\right)\left(\partial_{z} \phi\right)\right\} \cdots \text { (iii) }
\end{aligned}
$$


Next using (2.6) we have

$$
\begin{aligned}
\left(p_{1}^{0}-p^{0}\right)(\tilde{v})= & \frac{-m}{6}\left(\nabla_{\omega, v_{0}}^{1} \tilde{c}_{1}\left(\nabla_{T}^{1}\right)-\nabla_{\omega, v_{0}} \tilde{c}_{1}\left(\nabla_{T}\right)\right) \\
& -\frac{1}{2}\left(\nabla_{\omega, v_{0}}^{1}-\nabla_{\omega, v_{0}}\right) \tilde{c}_{1}\left(\nabla_{E}\right) \\
= & -\frac{m}{6} \nabla_{\omega, v_{0}}\left(\frac{i}{2 \pi} \bar{\partial}_{z} \partial_{z} \phi\right) \cdots \text { (i) } \\
& \left.+\frac{m}{6}\left(v_{0}\right\rfloor \partial \phi\right) \tilde{c}_{1}\left(\nabla_{T}\right) \cdots \text { (ii) } \\
& \left.+\frac{m}{6}\left(v_{0}\right\rfloor \partial \phi\right)\left(\frac{i}{2 \pi} \bar{\partial}_{z} \partial_{z} \phi\right) \cdots \text { (iii) } \\
& \left.+\frac{1}{2}\left(v_{0}\right\rfloor \partial \phi\right) \tilde{c}_{1}(E) \cdots \text { (iv) }
\end{aligned}
$$

From (2.3), using the commutation relation $\left.v\rfloor \int_{X / S}=\int_{X / S} v_{0}\right\rfloor$, the integrand for $-\left(\nabla_{Q, v}^{1}-\nabla_{Q, v}\right)$ can be expanded as (note that we only want the component in $\left.\Omega_{X / S}^{1,1}\right)$ :

$$
\begin{aligned}
& \left.\left.\frac{m}{6}\left(v_{0}\right\rfloor \partial \phi\right) \tilde{c}_{1}\left(\nabla_{T}\right)-\frac{m}{6} \partial_{z} \phi \wedge v_{0}\right\rfloor c_{1}\left(\nabla_{T}\right) \cdots \text { (i) } \\
& \left.\left.+\frac{m}{12}\left(v_{0}\right\rfloor \partial \phi\right) \frac{i}{2 \pi} \bar{\partial}_{z} \partial_{z} \phi-\frac{m}{12} \partial \phi \wedge v_{0}\right\rfloor \frac{i}{2 \pi} \bar{\partial}_{z} \partial \phi \cdots(\text { ii }) \\
& \left.\left.+\frac{1}{2}\left(v_{0}\right\rfloor \partial \phi\right) \tilde{c}_{1}\left(\nabla_{E}\right)-\frac{1}{2} \partial_{z} \phi \wedge v_{0}\right\rfloor c_{1}\left(\nabla_{E}\right) \cdots \text { (iii) }
\end{aligned}
$$

We can now prove Proposition 2.1 through the following scheme of equalities. We denote by “”” equality modulo $\bar{\partial}_{z}$ exact forms in $\Omega_{X / S}^{1,1}$. First

$$
(2.10)(\mathrm{i}) \equiv(2.9)(\mathrm{ii})+(2.8)(\mathrm{i})+(2.9)(\mathrm{i}),
$$

where we have used the identity

$$
\left.\bar{\partial}_{z}\left(\nabla_{\omega, v_{0}}\left(\partial_{z} \phi\right)\right)=\nabla_{\omega, \bar{\partial}_{z} v_{0}}\left(\partial_{z} \phi\right)-\left(v_{0}\right\rfloor c_{1}\left(\nabla_{\omega}\right)\right)\left(\partial_{z} \phi\right)+\nabla_{\omega, v_{0}}\left(\bar{\partial}_{z} \partial_{z} \phi\right) .
$$

Next

$$
(2.10)(\mathrm{ii}) \equiv(2.8)(\mathrm{ii})+(2.9)(\mathrm{iii}) \text {, }
$$

and here one uses

$$
\left.\left.\left.\left.\bar{\partial}_{z}\left\{\left(v_{0}\right\rfloor \partial \phi\right) \partial_{z} \phi\right\}=\left(\bar{\partial}_{z} v_{0}\right\rfloor \partial \phi\right) \partial_{z} \phi-\left(v_{0}\right\rfloor \bar{\partial}_{z} \partial \phi\right) \partial_{z} \phi+\left(v_{0}\right\rfloor \partial \phi\right) \bar{\partial}_{z} \partial_{z} \phi
$$

Finally,

$$
(2.10)(\text { iii) })=(2.8)(\text { iii })+(2.9)(\text { iv })
$$

by using (2.7). 


\section{Identification of the Connections}

Let $\pi: X \rightarrow S$ be a proper smooth map of relative dimension one, then it is always locally Kähler [Bing]. This means that on sufficiently small open sets $U \subset S$, $\pi^{-1}(U)$ has a Kähler metric. However we assume $T_{X / S}$ is given a hermitian metric $g$ which needs not be the restriction of a Kähler metric on $\pi^{-1} U$. Let $h$ be a hermitian metric on $E, \nabla_{\mathrm{Q}}$ and $\nabla_{\mathrm{BS}}$ are the associated connections on $\lambda_{E}^{-1}$ and $\lambda_{E}$. The next proposition reduces the problem of showing $\nabla_{\mathbf{Q}}=-\nabla_{\mathrm{BS}}$ to a simpler context.

Proposition 3.1. It suffices to check $\nabla_{\mathrm{Q}}=-\nabla_{\mathrm{BS}}$ for families where $\chi\left(X_{s},\left.E\right|_{X_{s}}\right)=0$ and $\bar{\partial}_{z}: C^{\infty}\left(X_{s},\left.E\right|_{X_{s}}\right) \rightarrow C^{\infty}\left(X_{s},\left.E \otimes \Omega^{0,1}\right|_{X_{s}}\right)$ is invertible.

Proof. By taking the direct sum of $E$ with a suitable $F$, e.g. $F=E^{*} \otimes \omega$ we have $\chi\left(X_{s},\left.E \oplus F\right|_{X_{s}}\right)=0$. Assume that we can prove $\nabla_{\mathbf{Q}}^{E \oplus F}=-\nabla_{\mathbf{B S}}^{E \oplus F}$ when the hermitian metric on $E \oplus F$ is direct sum of $h$ and any hermitian metric $h_{1}$ on $F$, then since $\lambda_{E \oplus F}=\lambda_{E} \otimes \lambda_{F}, \nabla_{\mathrm{Q}}^{E \oplus F}=\nabla_{\mathrm{Q}}^{E}+\nabla_{\mathrm{Q}}^{F}$ and likewise for $\nabla_{\mathrm{BS}}$. Since $h$ and $h_{1}$ are arbitrary we must have $\nabla_{\mathrm{Q}}^{E}=-\nabla_{\mathrm{BS}}^{E}, \nabla_{\mathrm{Q}}^{F}=-\nabla_{\mathrm{BS}}^{F}$. Thus we are reduced to showing $\nabla_{\mathrm{Q}}=-\nabla_{\mathrm{BS}}$ for bundles $E$ with index zero along fibers. Next we reduce to the case where $\bar{\partial}_{z}$ is invertible. The problem is local in $S$; it suffices to show $\nabla_{\mathrm{Q}}=-\nabla_{\mathrm{BS}}$ in the neighborhood of a given point $s_{0} \in S$.

Let $A$ be the (infinite dimensional) affine space which parametrizes the complex structures on a vector bundle over a Riemann surface (cf. [Q]). Over the moduli space of Riemann surfaces of genus $g$ let $\mathscr{E} \stackrel{P}{\longrightarrow} \mathscr{M}_{g}$ be the fiber space with fibers isomorphic to $A$ and whose local sections over $\mathscr{M}_{g}$ parametrize holomorphic vector bundles over families of Riemann surfaces. The family $E \rightarrow X_{s}, s \in U$ corresponds to a holomorphic $\operatorname{map} f: U \rightarrow \mathscr{E} P f(s)=\left\{X_{s}\right\}$. We want to extend $E$ to a vector bundle $\widetilde{E}$ over the family $X_{s} \times D_{\varepsilon} \rightarrow U \times D_{\varepsilon}$, where $D_{\varepsilon} \subset \mathbb{C}$ is a disc of radius $\varepsilon$ around 0 such that $\left.\widetilde{E}\right|_{\left(X_{s}, 0\right)}=E$ and $\bar{\partial}_{z}$ acting on $\left.\widetilde{E}\right|_{\left(X_{s}, t\right)}$ is invertible for $t \neq 0$. This corresponds to extending $f$ to a holomorphic map $\tilde{f}: U \times D_{\varepsilon} \rightarrow \mathscr{E}$, $\tilde{f}(s, 0)=f(s)$ and such that $\tilde{f}(s, t)$ satisfies the above requirement. The condition $\left.E\right|_{X_{s}}$ has index zero implies there is an open dense set in $A$ where $\bar{\partial}_{z}$ is invertible, thus we can find such extensions $\tilde{f}$.

Now consider such a family over $U \times D_{\varepsilon}$. The set where $\bar{\partial}_{z}$ is invertible is an open dense subset. Let the metrics be extended to this family. If we know $\nabla_{\mathrm{Q}}=-\nabla_{\mathrm{BS}}$ on the open set where $\bar{\partial}_{z}$ is invertible then by continuity we conclude that $\nabla_{\mathrm{Q}}=-\nabla_{\mathrm{BS}}$ on $U$. This finishes the proof.

From now on we assume $E \rightarrow X$ satisfies the conditions in Proposition 3.1. Let $G(z, \zeta)$ be the Schwartz kernel of the inverse $\bar{\partial}_{z}^{-1}$. Then the condition $\bar{\partial}_{z} \bar{\partial}_{z}^{-1}=1$ is equivalent to

$$
\bar{\partial}_{(z, \zeta)} G(z, \zeta)=\Delta \text { as distributions. }
$$

We may consider $G(z, \zeta)$ as a parametrix and (3.1) implies that $\bar{\partial}_{(z, \zeta)} G(z, \zeta)=0$ as a smooth form extended across $\Delta$. Let $\mathscr{B}_{E} \subset E \otimes E^{\prime}(2 \Delta)$ be the subsheaf with pole along $\Delta$ of order 2 or less and which by residue map $R$ has image in $\mathscr{A}_{E / S}$. There is an exact sequence

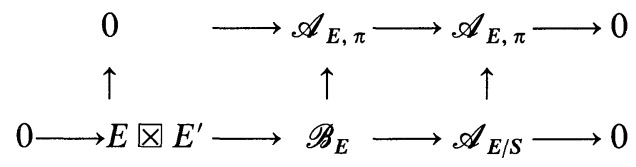


and given $u \in \mathscr{A}_{E / S}, G(u)=u_{z} G(z, \zeta) \in \mathscr{B}_{E}$ gives a splitting of (3.2). There is a natural projection $\mathscr{B}_{E} \vec{\beta}^{\text {tr }} \mathscr{A}_{E}^{-1}$. We can use $(\beta \circ G, 0)$ in place of $\left(p^{-1}, p^{0}\right)$ in Sect. 1 to get a splitting of (1.2).

Lemma 3.2. The connection on $\lambda_{E}$ corresponding to the splitting $(\beta \circ G, 0)$ is integrable.

Proof. Under the hypothesis $R^{0} \pi_{*} E=R^{1} \pi_{*} E=0$ we have $R^{0} \pi_{*}\left(\mathscr{B}_{E}-\mathscr{A}_{E, \pi}\right)$ $\cong \mathscr{T}_{S}$ and by [BS 2.3.2(iii)], $\nabla=R^{0} \pi_{*}(\beta \circ G)$ is the integrable connection.

Using (1.10) and Lemma 3.2 we express the difference of the Beilinson-Schechtman connection and the integrable connection as fiber integral of $C^{\infty}$ forms,

$$
\nabla_{\mathrm{BS}, v}-\nabla_{v}=\int_{X / S}\left(p^{-1}-G\right)\left(\bar{\partial}_{z} \tilde{v}\right)+p^{0}(\tilde{v}) .
$$

Lemma 3.3. Suppose $g_{1}$ is a hermitian metric on $T_{X / S}$ which is the restriction of a Kähler metric on $T_{X} \mid \pi^{-1}(U)$. Let $v_{0}$ be the lift of $v$ to $T_{X}$ which is orthogonal to $T_{X / S}$ for the Kähler metric, and $\tilde{v}=\nabla_{E, v_{0}}$, then

$$
\int_{x / S} p^{0}(\tilde{v})=0
$$

Proof. With the choice $\tilde{v}=\nabla_{E, v_{0}}, \pi_{\nabla_{E}}\left(v_{0}\right)=0$. So by (1.4),

$$
\int_{X / S} p^{0}(\tilde{v})=\frac{m}{6} \int_{X / S} \nabla_{\omega, v_{0}}\left(\tilde{c}_{1}\left(\nabla_{T}^{1}\right)\right)+\frac{1}{2} \int_{X / S} \nabla_{\omega, v_{0}}\left(\tilde{c}_{1}\left(\nabla_{E}\right)\right) .
$$

Let $\omega_{z}$ be the Kähler form along the fibers arising from $g_{1}$, then by [BFI, (1.8), BGS II, Th. 1.14]

$$
\begin{aligned}
v \int_{X / S} \tilde{c}_{1}\left(\nabla_{E}\right) & =v \int_{X / S}\left\langle\tilde{c}_{1}\left(\nabla_{E}\right), \omega_{z}\right\rangle \omega_{z} \\
& =\int_{X / S}\left\{\left\langle\nabla_{\omega, v_{0}} \tilde{c}_{1}\left(\nabla_{E}\right), \omega_{z}\right\rangle+\left\langle\tilde{c}_{1}\left(\nabla_{E}\right), \nabla_{\omega, v_{0}} \omega_{z}\right\rangle\right\} \omega_{z} .
\end{aligned}
$$

$\int_{X / S} \tilde{c}_{1}\left(\nabla_{E}\right)$ is constant in $s$ and $\nabla_{\omega, v_{0}} \omega_{z}=0$. Hence by (3.5) $\int_{X / S} \nabla_{\omega, v_{0}} \tilde{c}_{1}\left(\nabla_{E}\right)=0$. In the same way the other integral in right hand-side of (3.4) vanishes.

We consider next the connection form for $\nabla_{\mathrm{Q}}$, computed with respect to the metrics $g_{1}$ and $h . v_{0}$ will denote the horizontal lift prescribed in Lemma 3.3. $\lambda_{E}^{-1}$ has a canonical holomorphic section $T(\bar{\partial})$ nonzero over the points where $\bar{\partial}_{z}$ is invertible. With respect to the section $T(\bar{\partial}), \nabla_{\mathrm{Q}}$ has connection 1 form which is the $(1,0)$ component of (cf. [BGS III, (1.43), (1.48), (1.63)])

$$
\delta_{0}=\int_{0}^{\infty} \operatorname{Tr}_{s}\left[\exp \left(-u D^{2}\right)(\tilde{\nabla} D) D\right] d u,
$$

where $D=\bar{\partial}_{z}+\bar{\partial}_{z}^{*}$ and $\tilde{\nabla}_{v}=\nabla_{E, v_{0}}+\nabla_{T}$ is considered as a connection on an infinite dimensional bundle. By [BFI, Th. 1.15] this may be expressed as

$$
\delta_{0}=\lim _{t \rightarrow 0} \operatorname{Tr}_{s}\left[\exp \left(-t D^{2}\right) D^{-1}(\tilde{\nabla} D)\right] .
$$


Since $\bar{\partial}_{z}^{*} \circ \bar{\partial}_{z}^{-1}=0$ we have

$$
D\left(\bar{\partial}_{z}^{-1} \circ \tilde{\nabla} \bar{\partial}_{z}\right)=\tilde{\nabla} \bar{\partial}_{z} .
$$

Hence $\bar{\partial}_{z}^{-1} \circ \tilde{\nabla} \bar{\partial}_{z}=D^{-1} \tilde{\nabla} \bar{\partial}_{z}$, and since $\tilde{\nabla}_{v} \bar{\partial}^{*}=0$ by [BGS II, (1.51)] we have

$$
D^{-1}\left(\tilde{\nabla}_{v} D\right)=\bar{\partial}_{z}^{-1}\left(\tilde{\nabla}_{v} \bar{\partial}_{z}\right) \text {. }
$$

Next from [B, Th. 2.5] $\tilde{\nabla} \bar{\partial}_{z}=\tilde{\nabla} \circ \bar{\partial}_{z}+\bar{\partial}_{z} \circ \tilde{\nabla}$, hence

$$
\tilde{\nabla}_{v} \bar{\partial}_{z}=\tilde{\nabla}_{v} \circ \bar{\partial}_{z}-\bar{\partial}_{z} \circ \tilde{\nabla}_{v}=-\bar{\partial}_{z} \tilde{v}
$$

We now have

$$
\begin{aligned}
\delta_{0}(v)= & -\lim _{t \rightarrow 0} \operatorname{Tr}_{s}\left[\exp \left(-t D^{2}\right) D^{-1}\left(\tilde{\nabla}_{v} D\right)\right] \\
= & \lim _{t \rightarrow 0} \operatorname{Tr}_{s}\left[\exp \left(-t D^{2}\right) \bar{\partial}_{z}^{-1} \circ\left(\bar{\partial}_{z} \tilde{v}\right)\right] \\
= & \lim _{t \rightarrow 0} \operatorname{Tr}_{s}\left[\exp \left(-t D^{2}\right)\left(\bar{\partial}_{z}^{-1}-p^{-1}\right)\left(\bar{\partial}_{z} \tilde{v}\right)\right] \\
& +\lim _{t \rightarrow 0} \operatorname{Tr}_{s}\left[\exp \left(-t D^{2}\right) p^{-1}\left(\bar{\partial}_{z} \tilde{v}\right)\right] .
\end{aligned}
$$

In Sect. 4 we shall prove

\section{Proposition 3.4.}

$$
\lim _{t \rightarrow 0} \operatorname{Tr}_{s}\left[\exp \left(-t D^{2}\right) p^{-1}\left(\bar{\partial}_{z} \tilde{v}\right)\right]=0
$$

Given this proposition and the fact that $\bar{\partial}_{z}^{-1}-p^{-1}$ has a smooth kernel it follows from (3.3), (3.10) and Lemma 3.3 that $\nabla_{\mathrm{Q}}=-\nabla_{\mathrm{BS}}$ when the connections are defined in terms of the metrics $g_{1}$ and $h$. But then by Proposition $2.1 \nabla_{\mathrm{Q}}=-\nabla_{\mathrm{BS}}$ for any hermitian metrics $g$ and $h$.

Theorem 3.5. Let $\pi: X \rightarrow S$ be a proper smooth map of relative dimension one. Let $g$, $h$ be hermitian metrics on $T_{X / S}$ and $E$ and let $\nabla_{\mathrm{Q}}$ and $\nabla_{\mathrm{BS}}$ be the connections on $\lambda_{E}^{-1}$ and $\lambda_{E}$ respectively. Then $\nabla_{\mathrm{BS}}=-\nabla_{\mathrm{Q}}$.

\section{Calculations Involving the Heat Kernel}

We can restate Proposition 3.4 as

$$
\lim _{t \rightarrow 0} \operatorname{Tr}\left[e^{-t \Delta} p^{-1}\left(\bar{\partial}_{z} \tilde{v}\right)\right]=0
$$

where $\Delta=\bar{\partial}_{z}^{*} \bar{\partial}_{z}$. Reecall that in local coordinates

$$
\begin{aligned}
\tilde{v} & =\frac{\partial}{\partial s}+a(z) \frac{\partial}{\partial z}+B(z), \\
\bar{\partial}_{z} \tilde{v} & =\frac{\partial a}{\partial \bar{z}} d \bar{z} \frac{\partial}{\partial z}+\bar{\partial}_{z} B(z) .
\end{aligned}
$$


Lemma 4.1. The kernel function of $e^{-t \Delta} \circ p^{-1}\left(\bar{\partial}_{z} \tilde{v}\right)$ is given by

$$
\frac{\partial}{\partial z}\left(\bar{\partial}_{z} a \int_{y} e^{-t \Delta}(x, y) p(y, z) d v_{y}\right)+\left(\int_{y} e^{-t \Delta}(x, y) p(y, z) d v_{y}\right) \bar{\partial}_{z} B(z) .
$$

Proof. Let $\varphi$ be a smooth section of $E$ with compact support. Recall by (1.3) that $p(y, z)$ is a 1 form in $d z$,

$$
\begin{aligned}
& \left(e^{-t \Delta} \circ p^{-1 \circ \bar{\partial}_{z}} \tilde{v}\right) \varphi(x) \\
& =\int_{y} \int_{z} e^{-t \Delta}(x, y) p(y, z)\left\{\bar{\partial}_{z} a \frac{\partial \varphi}{\partial z}+\left(\bar{\partial}_{z} B\right) \varphi(z)\right\} d v_{y} \\
& =\int_{z} \frac{\partial}{\partial z}\left(\bar{\partial}_{z} a \int_{y} e^{-t \Delta}(x, y) p(y, z) d v_{y}\right) \varphi(z) \\
& \quad+\int_{z}\left(\int_{y} e^{-t \Delta}(x, y) p(y, z) d v_{y}\right)\left(\bar{\partial}_{z} B\right) \varphi(z) .
\end{aligned}
$$

Clearly then (4.1) will follow by showing:

(i) $\left.\lim _{t \rightarrow 0} \frac{\partial}{\partial z} \int_{y} e^{-t \Delta}(x, y) p(y, z) d v_{y}\right|_{z=x}=0$,

(ii) $\left.\lim _{t \rightarrow 0} \int_{y} e^{-t \Delta}(x, y) p(y, z) d v_{y}\right|_{z=x}=0$.

We shall first prove (4.2) (i). Consider the asymptotic expansion of $e^{-t \Delta}$ (cf. [P]),

$$
e^{-t \Delta}=\frac{1}{2 \pi t} e^{-\frac{d^{2}}{2 t}}\left(U_{0}+t U_{1}+\ldots\right)
$$

where $d$ is the distance function. We set in local coordinates $x=0$, and make the change of variable $y-z=r e^{i \theta}$. Note that since $\lim _{t \rightarrow 0} e^{-t \Delta}(x, y)$ is the delta function at $x$ we need only consider the integrals of (4.2) for $y$ in coordinate neighborhoods around $x$. Using (1.3) and the substitution for $y$, the limit in (4.2) (i) breaks up into a sum of the following types (omitting some constant factors):

$$
\begin{aligned}
& \text { (i) }\left.\lim _{t \rightarrow 0} \int \frac{1}{t^{2}} e^{-\frac{d^{2}}{2 t}}\left(\frac{\partial}{\partial z} d^{2}\right)\left(U_{0}+t U_{1}+\ldots\right) \frac{1}{r e^{i \theta}} d v_{y}\right|_{z=0}, \\
& \text { (ii) }\left.\lim _{t \rightarrow 0} \int \frac{1}{t^{2}} e^{-\frac{d^{2}}{2 t}} \frac{\partial}{\partial z}\left(U_{0}+t U_{1}+\ldots\right) \frac{1}{r e^{i \theta}} d v_{y}\right|_{z=0}, \\
& \text { (iii) }\left.\lim _{t \rightarrow 0} \frac{\partial}{\partial z} \int e^{-t \Delta} \sum_{i \geqq 0} A_{i}(y)(z-y)^{i} d v_{y}\right|_{z=0} .
\end{aligned}
$$

Lemma 4.2. (i) There exist complex geodesic coordinates y at 0 satisfying

$$
\frac{\partial g_{y \bar{y}}(0)}{\partial y}=\frac{\partial g_{y \bar{y}}(0)}{\partial \bar{y}}=\frac{\partial^{2} g_{y \bar{y}}(0)}{(\partial y)^{2}}=\frac{\partial^{2} g_{y \bar{y}}(0)}{(\partial \bar{y})^{2}}=0 \text {. }
$$


(ii) For any complex coordinate $y$ at 0 , there exists a local frame $\left\{e_{1}, \ldots, e_{m}\right\}$ of E near 0 such that

$$
\frac{\partial h_{i j}(0)}{\partial y}=\frac{\partial h_{i j}(0)}{\partial \bar{y}}=\frac{\partial^{2} h_{i j}(0)}{(\partial y)^{2}}=\frac{\partial^{2} h_{i j}(0)}{(\partial \bar{y})^{2}}=0 .
$$

Proof. Although the proofs are similar, the statements here are slightly more than what is available in standard references (cf. [W, p. 83)]. We will thus prove (ii); the same argument works for (i). It is standard that there exists a frame $\left\{e_{1}, \ldots, e_{m}\right\}$ at 0 such that $h_{i j}(0)=\delta_{i \bar{j}}$ and $d h(0)=0$. Consider

$$
\tilde{e}_{i}=e_{i}+\sum_{j} a_{i j} z^{2} e_{j}
$$

Then

$$
\begin{aligned}
\tilde{h}_{i j} & =\left\langle\tilde{e}_{i}, \tilde{e}_{j}\right\rangle=\left\langle e_{i}+\sum_{\alpha} a_{i \alpha} z^{2} e_{\alpha}, e_{j}+\sum_{\beta} a_{j \beta} z^{2} e_{\beta}\right\rangle \\
& =h_{i j}+\sum_{\alpha} a_{i \alpha} z^{2} h_{\alpha \bar{j}}+\sum_{\beta} \bar{a}_{j \beta} \bar{z}^{2} h_{i \bar{\beta}}+0\left(|z|^{4}\right) .
\end{aligned}
$$

By using $h_{i j}(0)=\delta_{i \bar{j}}$ and $d h(0)=0$ we have

$$
\tilde{h_{i j}}(0)=\delta_{i \bar{j}} \text { and } d \tilde{h}(0)=0 \text {. }
$$

Further $\frac{\partial^{2}}{\partial z^{2}} \tilde{h_{i j}}(0)=\frac{\partial^{2} h_{i \bar{j}}}{\partial z^{2}}(0)+a_{i j}$. Hence to make $\frac{\partial^{2}}{\partial z^{2}} \tilde{h_{i j}}(0)=0$ it is equivalent to choosing $a_{i j}=-\frac{\partial^{2} h_{i j}}{\partial z^{2}}(0)$.

Using Lemma 4.2 and the expansion formula for the distance function [D, (2.2)]:

$$
d^{2}\left(0, z+r e^{i \theta}\right)=\left|z+r e^{i \theta}\right|^{2}+\text { const }\left|z+r e^{i \theta}\right|^{4}+\mathrm{O}\left(r^{5}\right) .
$$

It follows that

$$
\left.\frac{\partial}{\partial z} d^{2}\right|_{z=0}=r e^{-i \theta}+\text { const } r^{3} e^{-i \theta}+\mathrm{O}\left(r^{4}\right)
$$

Also it follows from $[\mathrm{P},(4.9)]$ that

$$
U_{0}(0, y)=I+M\left|z+r e^{i \theta}\right|^{2}+\mathrm{O}\left(r^{3}\right),
$$

where $M$ is a constant matrix.

Lemma 4.3. $\lim _{t \rightarrow 0} \int \frac{1}{t^{2}} e^{-\frac{d^{2}}{2 t}} \frac{\mathrm{O}\left(r^{4}\right)}{r e^{i \theta}} d v_{y}=0$.

Proof. Use polar coordinates $d v_{y}=g_{r \bar{r}} r d r d \theta$ and change variable $r=\sqrt{t} \tilde{r}$, $d=\sqrt{t} \tilde{d}$; the above integral becomes

$$
\frac{1}{t^{2}} \int e^{-\frac{1}{2} \tilde{d}^{2}} \cdot \frac{t^{2} \mathrm{O}\left(\tilde{r}^{4}\right)}{e^{i \theta}}(\sqrt{t}) d \tilde{r} d \theta=\mathrm{O}(\sqrt{t}),
$$

which proves the lemma. 
By (4.5) and Lemma 4.3 we can reduce (4.3) (i) to

$$
\lim _{t \rightarrow 0} \int \frac{1}{t^{2}} e^{-\frac{d^{2}}{2 t}}\left(r e^{-i \theta}+c r^{3} e^{-i \theta}\right)\left(U_{0}+t U_{1}\right) \frac{1}{r e^{i \theta}} d v_{y},
$$

where $t^{2} U_{2}+\ldots$ is dropped by the same argument as in Lemma 4.3. We consider the most singular term in (4.7), the other terms can be treated in the same way,

$$
\begin{aligned}
\lim _{t \rightarrow 0} & \int \frac{1}{t^{2}} e^{-\frac{d^{2}}{2 t}} U_{0} e^{-2 i \theta} d v_{y} \\
= & \lim _{t \rightarrow 0} \int \frac{1}{t^{2}} e^{-\frac{d^{2}}{2 t}}\left(I+M r^{2}+\mathrm{O}\left(r^{3}\right)\right) e^{-2 i \theta} g_{r \bar{r}} r d r d \theta \\
\quad= & \lim _{t \rightarrow 0} \int \frac{1}{t^{2}} e^{-\frac{1}{2}\left(\tilde{r}^{2}+t \tilde{r}^{4}+t^{\frac{3}{2}} \mathrm{O}\left(\tilde{r}^{5}\right)\right)}\left(I+M t \tilde{r}^{2}+t^{\frac{3}{2}} \mathrm{O}\left(\tilde{r}^{3}\right)\right) e^{-2 i \theta} t \tilde{r} d \tilde{r} d \theta
\end{aligned}
$$

where we made the change of variable $r=\sqrt{t} \tilde{r}$ in the last integral.

Lemma 4.4. $\lim _{t \rightarrow 0} \frac{1}{t^{2}} \int e^{-\frac{1}{2}\left(\tilde{r}^{2}+t \tilde{r}^{4}\right)}\left(I+M t \tilde{r}^{2}+t^{\frac{3}{2}} \mathrm{O}\left(\tilde{r}^{3}\right)\right) e^{-2 i \theta} t \tilde{r} d \tilde{r} d \theta=0$.

Proof. The term involving $\mathrm{O}\left(\tilde{r}^{3}\right)$ vanishes as in Lemma 4.3. The remaining terms vanish when we integrate in $\theta$. to

$$
\text { Expanding } e^{\left(t^{\frac{3}{2}} \mathrm{O}\left(\tilde{r}^{5}\right)\right)}=1+t^{\frac{3}{2}} \mathrm{O}\left(\tilde{r}^{5}\right)+\mathrm{O}\left(t^{2}\right) \text { the last integral in (4.8) is reduced }
$$

$$
\lim _{t \rightarrow 0} \frac{1}{t^{2}} \int e^{-\frac{1}{2}\left(\tilde{r}^{2}+t \tilde{r}^{4}\right)}\left(t^{\frac{3}{2}} \mathrm{O}\left(\tilde{r}^{5}\right)+\mathrm{O}\left(t^{2}\right)\right)\left(I+M+t \tilde{r}^{2}+\ldots\right) e^{-2 i \theta} t \tilde{r} d \tilde{r} d \theta=0(4.9)
$$

This proves the vanishing of (4.3) (i). Similarly using (4.6) instead of (4.5) one can show the vanishing of (4.3) (ii). Finally to evaluate (4.3) (iii) we do not make the substitution $y=z+r e^{i \theta}$. Differentiating $\frac{\partial}{\partial z}$ in the integrand of (4.3) (iii) we have

$$
\lim _{t \rightarrow 0} \int e^{-t \Delta}(0, y)\left(A_{1}(y)+\mathrm{O}(|y|)\right) d v_{y} .
$$

By (1.3) and Lemma (4.2)

$$
A_{1}(y)=A_{1}(0)+\mathrm{O}(|y|)=0+\mathrm{O}(|y|) .
$$

Hence (4.10) reduces to

$$
\lim _{t \rightarrow 0} \int e^{-t \Delta}(0, y) \mathrm{O}(|y|) d v_{y}=0 .
$$

This finishes the proof of (4.2) (i). Finally note that the proof of (4.2) (ii) is much simpler, e.g. expanding $p(y, z)$ in two parts the vanishings are already covered in the vanishings of (4.3) (ii) and (iii). We have therefore proved (4.1).

\section{References}

[BS] Beilinson, A., Schechtman, V.: Determinant bundles and Virasoro algebras. Commun. Math. Phys. 188, 651-701 (1988) 
[Bing] Bingener, J.: On deformations of Kähler spaces II. Archiv der Mathematik 41, 517-530 (1983)

[B] Bismut, J M.: The Atiyah-Singer Index theorem for families of Dirac operators Invent. Math. 83, 91-151 (1986)

[BF] Bismut, J M., Freed, D.S : The analysis of elliptic families I, II Commun Math Phys. 106, 159-176 (1986); 107, 103-163 (1986)

[BGS] Bismut, J.M., Gillet, H., Soulé, C.: Analytic torsion and holomorphic determinant bundles, I, II, III Commun Math Phys 115, 49-126, 301-351 (1988)

[Bt C] Bott, R., Chern, S.S.: Hermitian vector bundles and the equidistribution of the zeros of their holomorphic sections. Acta Math. 114, 71-112 (1968)

[D] Donnelly, H.: Spectrum and the Fixed Point Sets of Isometrics, I. Math. Ann. 224, 161-170 (1976)

[P] Patodi, V K : An analytic proof of Riemann-Roch-Hirzebuch theorem for Kähler manifolds. J. Diff Geom. 5, 251-283 (1971)

[Q] Quillen, D.: Determinants of Cauchy-Riemann operators over a Riemann surface. Funct. Anal. Appl. 19, 31-34 (1985)

[TT] Toledo, D., Tong, Y.L : A parametrix for $\bar{\partial}$ and Riemann Roch in Cech Theory. Topology 15, 273-301 (1976)

[T] Tong, Y.L: Connections on determinant bundles J of Algebraic Geometry 2, 443-486 (1993)

[W] Wells, R.O.: Differential analysis on complex manifolds. Englewood Cliffs, NJ: PrenticeHall 1973

Communicated by A. Jaffe 
